Bundesgesundheitsbl 2017 · 60:532-536

DOI 10.1007/s00103-017-2534-9

Online publiziert: 27. März 2017

(c) Der/die Autor(en) 2017. Dieser Artikel ist eine Open-Access-Publikation.

CrossMark

Magdalena María Marx - Franzisca Marie Dulas • Katja Maria Schumacher

Medizinische Klassifikationen, Deutsches Institut für Medizinische Dokumentation und Information, Köln, Deutschland

\title{
Verbesserung der Sichtbarkeit seltener Erkrankungen in Gesundheitssystemen durch spezifische Routinekodierung
}

kungen werden vor allem geschätzt [3, $4]$.

nur in Deutschland eine wesentliche Aufgabe der Leistungserbringer im Gesundheitswesen. Die Kodes, die bei der Verschlüsselung von Diagnosen dokumentiert werden, sind Grundlage mehrerer Prozesse im Gesundheitssystem. Unter anderem sind die Steuerung der Vergütung, die Vorgänge der Qualitätssicherung, die Erstellung von amtlichen Statistiken und des Risikostrukturausgleichs sowie in vielen Fällen die epidemiologische und klinische Forschung in diesen Kodes in Deutschland verankert. Die Informationen, welche durch die Auswertung dieser Kodes gewonnen werden, werden auch von den Entscheidungsträgern u. a. für Zwecke der Planung, Berichterstattung, Evaluation und Analyse von gesundheitspolitischen Maßnahmen genutzt.

Im Bereich der seltenen Erkrankungen stellt die eindeutige Kodierung weltweit eine große Herausforderung dar [1, 2, 13]. Die Abbildung seltener Erkrankungen in den Klassifikationen, die für die Erfassung von Routinedaten eingesetzt werden, ist nicht ausreichend. Ein großer Anteil dieser Erkrankungen ist in diesen Klassifikationen nicht oder nicht spezifisch erfasst. Dementsprechend ist die statistische Abbildung seltener Erkrankungen und die darauf basierende Einschätzung ihrer Bedeutung in Gesundheitssystemen bisher nicht zufriedenstellend $[1,2]$. Gesicherte epidemiologische Daten liegen zurzeit nur partiell vor und die Zahlen zu seltenen Erkran-

\section{Die Routinekodierung seltener Erkrankungen anhand ICD-10}

Die „Internationale statistische Klassifikation der Krankheiten und verwandter Gesundheitsprobleme, 10. Revision, German Modification" (ICD-10-GM) ist die amtliche Klassifikation zur Verschlüsselung von Diagnosen in der ambulanten und stationären Versorgung in Deutschland. Die ICD-10-GM ist eine Adaptation der „Internationalen statistischen Klassifikation der Krankheiten und verwandter Gesundheitsprobleme, 10. Revision“" (ICD-10) der Weltgesundheitsorganisation (WHO). Sie ist eine an die Erfordernisse des deutschen Gesundheitswesens angepasste Fassung. In Aufbau und Struktur entspricht die ICD-10-GM der Systematik der ICD-10 der WHO [5]. Die ICD-10 der WHO ist weitgehend die Klassifikation, die für die Erfassung der Mortalität sowie der Morbidität in den europäischen Ländern angewandt wird.

Die Abbildung einzelner seltener Erkrankungen mittels der ICD-10 ist nicht eindeutig. Nur wenige dieser Erkrankungen sind mit der ICD-10 spezifisch kodierbar, haben also einen eigenen Kode $[1,6]$. Im Jahr 2015 hatten bspw. nur ca. 355 seltene Erkrankungen (von insgesamt mehr als 8000) einen eigenen spezifischen ICD-10-Kode [7]. Bis heute hat sich diese Anzahl nicht wesentlich verändert. Eine Vielzahl seltener Erkrankungen wird in der ICD-10 unspezifi- schen Kodes zugeordnet (als sog. Inklusiva) und ist deshalb anhand des ICD10-Kodes statistisch nicht eindeutig zu erfassen. Eine Erweiterung der Granularität der ICD-10 bis hin zu den einzelnen seltenen Erkrankungen ist nicht vorgesehen. Die grobe Unterteilung der ICD-10 ist für ihre Zwecke ausreichend und soll im Sinne der Vermeidung von Bürokratie auch nicht weiter verfeinert werden.

\section{Die Alternative: eine doppelte Kodierung}

Um die Sichtbarkeit von seltenen Erkrankungen in Gesundheitssystemen zu verbessern, bieten sich aber auch andere Lösungen an, als die ICD-10 zu verändern. Die doppelte Kodierung seltener Erkrankungen anhand eines ICD-10-Kodes und eines spezifischen Kodes für die jeweilige Erkrankung soll ihre eindeutige Verschlüsselung und somit ihre Identifizierung in den erfassten Daten ermöglichen.

Ein spezifisches Kodiersystem für seltene Erkrankungen bietet das europäische Referenzportal Orphanet (www. orpha.net), ein allgemein zugängliches europäisches Portal für Informationen über seltene Erkrankungen, welche sich auf Fachpublikationen begründen. Orphanet produziert eine Datenbank mit einem Verzeichnis seltener Erkrankungen, der sog. Orphanet-Nomenklatur. Mittlerweile ist die Orphanet-Nomenklatur in 7 verschiedenen Sprachen für die Öffentlichkeit verfügbar. Allen seltenen Erkrankungen in dieser Datenbank ist eine spezifische Kennnummer, die 
Ich finde, dass eine doppelte Kodierung der seltenen Erkrankungen anhand Orpha-

Kennnummer und ICD-10 in der Erfassung dazu beitragen kann, die seltenen

Erkrankungen im Gesundheitssystem besser sichtbar zu machen.

(36 Antworten)

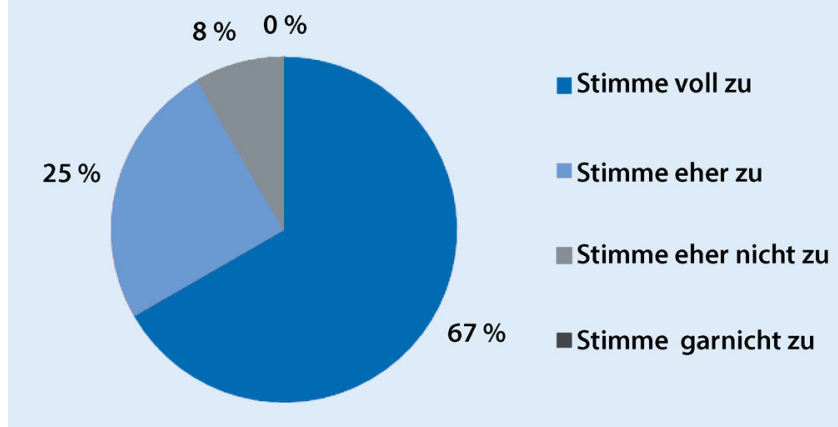

Abb. 1 ॥ Auszug aus der Onlineumfrage zur Evaluation des Projektes „Kodierung von Seltenen Erkrankungen" des DIMDI. Projektpartner, Kooperationspartner sowie Ansprechpartner unterschiedlicher Institutionen und Arbeitsgruppen aus dem Bereich seltener Erkrankungen wurden im März 2016 per E-Mail eingeladen an der Onlineumfrage teilzunehmen. Unter anderem wurden die Teilnehmer gebeten die Aussage in Abb. 1 im Likert-Skala-Verfahren zu bewerten

sog. Orpha-Kennnummer, zugeordnet [8]. Diese Orpha-Kennnummern sind wiederum in einem Klassifikationssystem geordnet.

Die doppelte Kodierung seltener Erkrankungen anhand des ICD-10-Kodes und der Orpha-Kennnummer stellt eine kurzfristige und ressourcenschonende Lösung dar. Zudem wird dadurch eine internationale Vergleichbarkeit ermöglicht. Diese Herangehensweise entspricht der Empfehlung des Rates der Europäischen Union für eine Maßnahme im Bereich seltener Krankheiten [2] und stößt auch auf eine große nationale Akzeptanz (• Abb. 1).

\section{Allein die Bereitstellung einer neuen Klassifikation ist nicht ausreichend}

Es gibt Faktoren, die eine standardisierte Diagnosekodierung seltener Erkrankungen anhand einer doppelten Kodierung gefährden könnten. Einerseits können folgende bekannte Faktoren die Interobservervariabilität bei der Kodierung erhöhen: dieverschiedenen Zwecke der Kodierung, der fachliche Hintergrund der Kodierer sowie das Vorhandensein eines finanziellen Antriebs (DRG-betriebene Gesundheitssysteme). Andererseits ha- ben seltene Erkrankungen üblicherweise eine multisystemische Ausprägung. Dies erhöht die Schwierigkeit der Kodierung bei der Verschlüsselung von Diagnosen anhand Klassifikationen, die, wie bei der ICD-10, größtenteils nach den Körpersystemen aufgebaut sind.

Um seltene Erkrankungen anhand der oben genannten doppelten Kodierung routinemäßig spezifisch zu verschlüsseln, soll außer dem ICD-10-Kode zusätzlich das System der Orpha-Kennnummern angewendet werden. Kodierer müssten also zwei voneinander unabhängige Systeme nutzen. Hierbei soll nicht außer net-Nomenklatur nicht ausschließlich für Zwecke der statistischen Erfassung von seltenen Erkrankungen konzipiert ist. Sie bietet bspw. auch Hilfestellung bei der Expertensuche.

All dies - Interobservervariabilität, multisystemische Ausprägung der seltenen Erkrankungen und Kodierung mit 2 Systemen - birgt das erhebliche Risiko, dass die beiden Systeme nicht einheitlich angewendet werden mit der Folge, dass die Daten statistisch nicht aussagefähig sind. Allein die Bereitstellung einer neuen Klassifikation ist nicht ausreichend, um valide Daten zu erheben. Dies wurde bereits in den Ländern, die bislang die OrAcht gelassen werden, dass die Orpha-
pha-Kennnummern für die Kodierung seltener Erkrankungen implementiert haben, beobachtet. Dort wurde der Bedarf an weiterer Unterstützung bei der Anwendung der Orphanet-Nomenklatur erkannt $[9,10]$. Dies könnte z. B. durch die Bereitstellung von Kodierrichtlinien erfolgen. Des Weiteren ist aus Sicht der Autoren eine sorgfältige Aufbereitung der Orphanet-Nomenklatur notwendig, um diese für die statistische Datenerhebung zu verwenden. Im besten Fall könnte dies durch die Aufbereitung eines Auszuges dieser Nomenklatur erfolgen. Dieser Auszug sollte nur die Daten, die für die Routinekodierung sinnvoll bzw. notwendig sind, enthalten.

\section{Weitere Herausforderungen der Kodierung seltener Erkrankungen}

Die geringe Anzahl von Patienten, die unter einer seltenen Erkrankung leiden, stellt eine Hürde dar, um mit gewöhnlichen Methoden epidemiologische Daten zu erheben und klinische Forschung zu betreiben [11]. Die Möglichkeit einer flächendeckenden Erfassung von Daten auch über Ländergrenzen hinweg ist für eine fundierte Forschung im Bereich seltener Erkrankungen von großer Bedeutung.

Die Anwendung der doppelten Kodierung seltener Erkrankungen anhand von Orpha-Kennnummern und ICD-10Kodes durch die EU-Länder würde den Datenaustausch und eine vereinfachte Patientenmobilität sowie eine umfassendere Forschung in diesem Raum ermöglichen. Infolgedessen würden sich die Chancen erhöhen, die Qualität der Behandlung der Patienten mit seltenen Erkrankungen zu verbessern.

Eine wichtige Herausforderung dieses potenziellen Datenaustausches ist die standardisierte Erfassung der Daten. Darüber hinaus ist die einheitliche und standardisierte Erfassung der Daten eine Voraussetzung, um Interoperabilität zu erreichen. Die Interoperabilität der Daten spielt eine große Rolle bei Maßnahmen im Bereich seltener Erkrankungen auf europäische Ebene, wie z. B. bei der Vernetzung der Leistungserbringer in den European Reference Networks (ERNs). 
Die Interoperabilität bestimmt in gewissem Maße den Umfang des Datenaustauschs auf dieser Ebene.

\section{Unternehmungen im Bereich Kodierung seltener Erkrankungen in Deutschland}

In Deutschland wird angestrebt, das Problem der Kodierung von seltenen Erkrankungen entsprechend dem Maßnahmenvorschlag 19 des „Nationalen Aktionsplans für Menschen mit Seltenen Erkrankungen" auf nationaler Ebene zu bewältigen [12]. Diese Maßnahme wird durch das Projekt „Kodierung von Seltenen Erkrankungen“ des Deutschen Instituts für Medizinische Dokumentation und Information (DIMDI) umgesetzt. Das wesentliche Ziel des DIMDIProjektes ist es, die Kodierung seltener Erkrankungen in Deutschland zu verbessern und insbesondere deren doppelte Kodierung anhand des ICD-10GM-Kodes (Kodes zur Verschlüsselung von Diagnosen in der ambulanten und stationären Versorgung) und der OrphaKennnummer zu vereinfachen.

In diesem vom Bundesministerium für Gesundheit geförderten Projekt wird ein sog. Musterdatensatz produziert, der es ermöglichen soll, die seltenen Erkrankungen in Deutschland anhand einer doppelten Kodierung spezifisch zu verschlüsseln [14]. Der Musterdatensatz soll die elektronische Kodierung so unterstützen, dass dabei keine zusätzlichen Schritte über die Routinekodierung mit der ICD-10-GM hinaus erforderlich werden. Die Implementierung des Musterdatensatzes soll durch eine eindeutige und einheitliche automatische Kodierung die standardisierte Verschlüsselung seltener Erkrankungen ermöglichen und dadurch zur Interoperabilität der Daten beitragen. Durch die Koppelung des Diagnosetextes mit dem jeweiligen Kode aus ICD-10-GM und aus der OrphanetNomenklatur wird es den Kodierern ermöglicht, bei Auswahl der Diagnose in einem Arbeitsschritt beide Kodes zu erhalten (s. - Tab. 1). Die Bereitstellung der Diagnosetexte jeweils mit den beiden Kodes erfolgt in einem seit vielen Jahren etablierten Format: mit der sog. AlphaID.

Bundesgesundheitsbl 2017·60:532-536 DOI 10.1007/s00103-017-2534-9

๑ Der/die Autor(en) 2017. Dieser Artikel ist eine Open-Access-Publikation.

\section{M. Marx · F. M. Dulas · K. M. Schumacher \\ Verbesserung der Sichtbarkeit seltener Erkrankungen in Gesundheitssystemen durch spezifische Routinekodierung}

\section{Zusammenfassung}

Die Auswertung von Routinedaten der Leistungserbringer des Gesundheitswesens ist eine wichtige Grundlage der Analyse, Planung sowie der Evaluation von gesundheitspolitischen Maßnahmen. Die Abbildung seltener Erkrankungen in den Klassifikationen, die für die Erfassung von Routinedaten eingesetzt werden, ist nicht ausreichend. Die eindeutige Kodierung seltener Erkrankungen stellt weltweit eine große Herausforderung dar. Es gibt bislang keine allgemeingültige internationale Lösung für die Routinekodierung dieser Erkrankungen.

Die doppelte Kodierung seltener Erkrankungen anhand des ICD-10-Kodes (International Classification of Diseases [ICD]) und der Orpha-Kennnummer stellt eine kurzfristige und ressourcenschonende alternative Lösung dar. Zudem wird eine internationale Vergleichbarkeit ermöglicht. Die spezifische Verschlüsselung seltener Erkrankungen durch die doppelte Kodierung kann ihre statistische Erfassung und dadurch ihre Abbildung in Gesundheitssystemen verbessern. Dennoch ist allein die Bereitstellung einer neuen Klassifikation nicht ausreichend, um valide Daten zu erheben. In Deutschland (sowie auf europäischer Ebene) sind bereits Maßnahmen eingeleitet worden, um die Implementierung dieser doppelten Kodierung zu unterstützen. Anschließend könnten möglicherweise basierend auf den besseren Daten - gezielt politische Maßnahmen eingeleitet werden, um eine bessere Versorgung der über 4 Mio. Menschen in Deutschland, die an einer seltenen Erkrankung leiden, gewährleisten zu können.

\section{Schlüsselwörter}

Kodierung seltener Erkrankungen · Interoperabilität · ICD-10 · Orpha-Kennnummer . Alpha-ID

\section{Improving the visibility of rare diseases in health care systems by specific routine coding}

\section{Abstract}

The evaluation of healthcare providers' routine data is an important basis for the analysis, planning and evaluation of measures in public health. The representation of rare diseases in the classifications that are used to record health data is not adequate. Coding rare diseases in a specific way is a challenge all around the world. There is still no general international solution for the routine coding of rare diseases.

The double coding of rare diseases with ICD10 Codes and Orphacodes is a short-term and low-cost alternative solution. Furthermore, this double coding enables international comparability. The specific encoding of rare diseases through this double coding can improve their capturing for statistical analysis and thus their visibility in healthcare systems. Nevertheless, the provision of a new classification is not enough to gather valid data. Some measures have already been adopted in Germany (and at the European level) in order to support the implementation of this double coding. Subsequently it would be possible to adopt more specific public health measures, based on better data, in order to provide better care to the more than four million people in Germany affected by rare diseases.

\section{Keywords}

Codification of rare diseases - Interoperability . RD-ACTION · Orphacode · Data sharing
Der Musterdatensatz ist auch Grundlage für die Durchführung anderer Projekte des Nationalen Aktionsplans für Menschen mit Seltenen Erkrankungen, wie z. B. dem Aufbau eines Registerprototyps oder dem Versorgungsatlas für Menschen mit seltenen Erkrankungen. Dennoch ist die Implementierung des
Musterdatensatzes bisher wenig verbreitet. Nur durch eine Implementierung dieser Datei in die Routinekodierung könnte in Erfahrung gebracht werden, ob diese Herangehensweise die Probleme der Kodierung seltener Erkrankungen in Deutschland lösen kann. 


\begin{tabular}{|c|c|c|c|c|c|}
\hline $\begin{array}{l}\text { Alpha-ID- } \\
\text { Kode }\end{array}$ & $\begin{array}{l}\text { ICD-10-GM- } \\
\text { Kode }\end{array}$ & $\begin{array}{l}\text { ICD-10-GM- } \\
\text { Sternkode }\end{array}$ & $\begin{array}{l}\text { ICD-10-GM- } \\
\text { Zusatzkode }\end{array}$ & $\begin{array}{l}\text { Orpha- } \\
\text { Kennnum- } \\
\text { mer }\end{array}$ & Eintrag \\
\hline |17291 & Q41.9 & - & - & 1201 & Dünndarmatresie \\
\hline 182889 & Q41.1 & - & - & 1201 & Apfelschalen-Syndrom \\
\hline 1118177 & Q41.1 & - & - & 1201 & Jejunalatresie \\
\hline 124937 & K90.8+ & M14.8* & - & 3452 & Whipple-Krankheit \\
\hline 15821 & K90.8 & - & - & 3452 & $\begin{array}{l}\text { Intestinale Lipodystro- } \\
\text { phie }\end{array}$ \\
\hline 132050 & M61.19 & - & - & 337 & $\begin{array}{l}\text { Fibrodysplasia ossificans } \\
\text { progressiva }\end{array}$ \\
\hline 181949 & M61.19 & - & - & 337 & Münchmeyer-Syndrom \\
\hline 19222 & Q87.8 & - & - & 912 & $\begin{array}{l}\text { Zerebrohepatorenales } \\
\text { Syndrom }\end{array}$ \\
\hline 1117676 & Q87.8 & - & - & 500 & $\begin{array}{l}\text { Kardiomyopathische } \\
\text { Lentiginose }\end{array}$ \\
\hline
\end{tabular}

Zusätzlich zum nationalen Projekt „Kodierung von Seltenen Erkrankungen“ beteiligt sich das DIMDI an einem internationalen Projekt, der Rare Disease Action (RD-Action). Das DIMDI leitet das Arbeitspaket 5 (WP 5) der RD-Action zur Steuerung, Pflege und Förderung der Einführung von OrphaKennnummern in EU-Mitgliedsstaaten. Durch die Arbeiten des Arbeitspakets 5 der RD-Action wird bereits auf europäischer Ebene versucht, Werkzeuge (Kodierrichtlinien, Prozesse und eine Masterdatei u. a.) zu entwickeln. Diese sollen die EU-Länder bei der Implementierung der Orphanet-Nomenklatur zur Routinekodierung unterstützen. Hierbei ist das DIMDI u. a. für das „Deliverable 5.3 A European integrated master file" und damit für den Entwurf der europäischen Masterdatei zur Kodierung verantwortlich. Die Teilnahme des DIMDI an dieser europäischen Arbeitsgruppe verwirklicht Synergien und ermöglicht Erfahrungsaustausch sowie die Kongruenz der nationalen Arbeiten und weiteren Entwicklungen mit den Arbeiten auf europäischer Ebene.

\section{Ausblick}

Für die Zukunft sind insbesondere die Weiterentwicklungen der WHO-Klassifikationen interessant, die diese doppelte Kodierung eventuell ersetzen bzw. direkt implementieren könnten. Es sind bereits Maßnahmen eingeleitet worden, die bei dem Revisionsprozess der ICD die Repräsentation seltener Erkrankungen in dieser Klassifikation verbessern sollen. Eine dieser Maßnahmen ist die Einrichtung einer sog. Topic Advisory Group (TAG) für seltene Erkrankungen unter der Leitung von Orphanet im Jahr 2009. Eine konkrete Lösung, um einzelne seltene Erkrankungen sichtbar zu machen, ist zwar strukturell entwickelt worden, aber bei dem jetzigen Entwicklungsstand (verfügbare Betaversion) der ICD-11 noch nicht voll $\mathrm{zu}$ erkennen. Die Herangehensweise des deutschen Projektes findet sich in dem für die ICD-11 angedachten Konzept für seltene Erkrankungen wieder: Über einen eindeutigen Identifikator soll jeder Diagnosetext in der ICD11 auffindbar sein und eine Verknüpfung $\mathrm{zu}$ anderen Klassifikationssystemen realisiert werden. Sollte dieses Konzept in der ICD-11 vollständig umgesetzt werden, so kann durch die aktuellen Arbeiten mit ICD-10-GM und Orpha-Kennnummern ein Umstieg auf die ICD-11 für diesen Bereich erleichtert werden.

Es gibt bislang keine allgemeingültige internationale Lösung für die Routinekodierung seltener Erkrankungen. Auch in Deutschland wurden in unterschiedlichen Kodierszenarien verschiedene Ansätze versucht. Dennoch sollen hier erste Schritte getan werden, wie bspw. die
Kodierprojekte des DIMDI, um einen möglichst gemeinsamen Weg zu finden, auswertbare Daten zu diesen Erkrankungen zu erfassen. Anschließend könnten möglicherweise, basierend auf den besseren Daten, gezielt politische Maßnahmen eingeleitet werden, um eine bessere Versorgung der über 4 Mio. Menschen in Deutschland, die an einer seltenen Erkrankung leiden, anbieten zu können.

\section{Fazit für die Praxis}

- Die Abbildung seltener Erkrankungen in den Klassifikationen, wie z. B. in der ICD-10, die bei der Erfassung von Routinedaten eingesetzt werden, ist nicht ausreichend.

- Die statistische Abbildung seltener Erkrankungen und die darauf basierende Einschätzung ihrer Bedeutung in Gesundheitssystemen sind bisher nicht zufriedenstellend.

- Die doppelte Kodierung seltener Erkrankungen anhand des ICD-10Kodes und der Orpha-Kennnummer von Orphanet stellt eine kurzfristige und ressourcenschonende Lösung dar, um ihre Sichtbarkeit zu verbessern.

- Die Bereitstellung einer neuen Klassifikation ist allein nicht ausreichend, um valide Daten zu erheben.

- Bei Maßnahmen im Bereich seltener Erkrankungen spielt die Interoperabilität der Daten eine große Rolle.

- In Deutschland wird durch die Testimplementierung des Musterdatensatzes im Rahmen eines DIMDI Projektes angestrebt, das Problem der Kodierung von seltenen Erkrankungen anhand einer standardisierten doppelten Kodierung auf nationaler Ebene zu bewältigen.

\section{Korrespondenzadresse}

\section{M. Marx}

Medizinische Klassifikationen, Deutsches Institut für Medizinische Dokumentation und Information

Waisenhausgasse 36-38a, 50676 Köln,

Deutschland

Magdalena.Marx@dimdi.de 


\section{Einhaltung ethischer Richtlinien}

Interessenkonflikt. M. M. Marx, F.M. Dulas und K. M. Schumacher sind beim Deutschen Institut für Medizinische Dokumentation und Information (DIMDI) tätig.

Dieser Beitrag beinhaltet keine von den Autoren durchgeführten Studien an Menschen oder Tieren.

Open Access. Dieser Artikel wird unter der Creative Commons Namensnennung 4.0 International Lizenz (http://creativecommons.org/licenses/by/4.0/deed. de) veröffentlicht, welche die Nutzung, Vervielfältigung, Bearbeitung, Verbreitung und Wiedergabe in jeglichem Medium und Format erlaubt, sofern Sie den/die ursprünglichen Autor(en) und die Quelle ordnungsgemäßnennen, einen Linkzur Creative Commons Lizenz beifügen und angeben, ob Änderungen vorgenommen wurden.

\section{Literatur}

1. The council of the European Union (2009) Council Recommendation of 8 June 2009 on an action in the field of rare diseases (2009/C 151/02). http:// eur-lex.europa.eu/LexUriServ.do?uri=0J:C:2009: 151:0007:0010:EN:PDF.Zugegriffen:04.Nov. 2016

2. CEGRD (2014) Recommendation on ways to Improve Codification for Rare Diseases in Health Information Systems. Adopted at the 3rd meeting of the Commission Expert Group on Rare Diseases (12.-13. November 2014). http://ec.europa.eu/ health/rare_diseases/docs/recommendation coding_cegrd_en.pdf.Zugegriffen:04.Nov. 2016

3. Schulz $M$, Wandrey $M$, Hering $R$, Schulz $M$, Bätzing-Feigenbaum J (2015) Prävalenz seltener Erkrankungen in der ambulanten Versorgung in Deutschland im Zeitraum 2008 bis 2011, Versorgungsatlas-Bericht Nr. 15/13, Bd. Zentralinstitut für die kassenärztliche Versorgung in Deutschland (Zi), 2016. Berlin http://www.versorgungsatlas. de/themen/alle-analysen-nach-datum-sortiert/? tab=6\&uid=63.Zugegriffen:04. Nov. 2016

4. Spielberg P (2001) Seltene Erkrankungen: „Das bisher Erreichte ist nur ein Tropfen auf dem heißen Stein". http://www.aerzteblatt.de/pdf/108/49/ a2643.pdf.Zugegriffen:04.Nov. 2016

5. DIMDI (2016) ICD-10-GM. http://www.dimdi.de/ static/de/klassi/icd-10-gm/index.htm. Zugegriffen:04.Nov. 2016

6. Robinson $P$ (2012) Classification and coding of rare diseases: overview of where we stand, rationale, whyitmatters and whatitcan change.http://www. ncbi.nlm.nih.gov/pmc/articles/PMC3504556/ pdf/1750-1172-7-S2-A10.pdf. Zugegriffen: 04. Nov. 2016

7. Aymé S, Bellet B, Rath A (2015) Rare diseases in ICD11: making rare diseases visible in health information systems through appropriate coding. http://ojrd.biomedcentral.com/articles/10.1186/ s13023-015-0251-8.Zugegriffen:04.Nov. 2016

8. Orphanet (2016) Orphadata. http://www. orphadata.org/cgi-bin/inc/product1.inc.php. Zugegriffen:04. Nov. 2016

9. WP5 (2015) RD-Action WP5 survey; Deliverable 5.1-part 1: Review existing technical implementations for RD coding. http://www.rd-action. eu/wp-content/uploads/2016/06/677024_D5.1 INTEGRATED_FINAL_2.pdf. Zugegriffen: 04 . Nov. 2016
10. WP5 (2016) Deliverable D5.1; Review document of existing technicalimplementations for rare disease coding. http://www.rd-action.eu/wp-content/ uploads/2016/06/677024_D5.1_INTEGRATED_ FINAL_2.pdf.Zugegriffen:04.Nov. 2016

11. Rückinger S, Boneberger A (2008) Epidemiologische Herausforderungen bei seltenen Krankheiten. Bundesgesundheitsbl Gesundheitsforsch Gesundheitsschutz 51:483-490

12. NAMSE (2013) Nationaler Aktionsplan für Menschen mit Seltenen Erkrankungen. http:// www.namse.de/images/stories/Dokumente/ nationaler_aktionsplan.pdf. Zugegriffen: 04 . Nov. 2016

13. W. A. Department of Health (2015) WA Rare Diseases Strategic framework 2015-2018. http://ww2.health.wa.gov.au/ /media/Files/ Corporate/Reports\%20and\%20publications/ PDF/Rare-diseases-strategic-framework.ashx. Zugegriffen:04.Nov. 2016

14. DIMDI (2015) Musterdatensatz. http://www.dimdi. de/dynamic/de/klassi/downloadcenter/alpha-id/ seltene-erkrankungen/alphaid_se_muster2016. zip.Zugegriffen: 26 . Juli 2016 